\author{
Military Technical College \\ Kobry El-Kobbah, \\ Cairo, Egypt
}

\author{
$9^{\text {th }}$ International Conference \\ on Electrical Engineering \\ ICEENG 2014
}

\title{
New Advanced IHS for Pansharpening of Hyperspectral Image
}

\author{
By \\ Mohamed Reda*, Ezz Eldin Farouk **
}

\begin{abstract}
$\underline{\text { Abstract: }}$
Image fusion techniques have been developed to utilize different types of image data. The aim of image fusion is to improve the quality of the information by combining two or more different types of images. Pan sharpening is one of the most common fusion techniques used for its efficiency and simplicity. It fuses a cube of multi/hyperspectral image of low spatial resolution with a panchromatic image (PAN) of a higher spatial resolution to obtain an image with high spectral and spatial resolution. This requires a transformation tool to separate the spatial information of the cube from its spectral information. Some of these transformations include Intensity-Hue-Saturation (IHS), principle component analysis (PCA), wavelet transform (WT) and independent component analysis (ICA). IHS is considered the most popular transformation tool because of its simplicity and efficiency. IHS introduces a fused image of high spatial resolution but it suffers from some spectral distortion. In this paper, we tried to solve this problem by using an adaptive image coefficient and a morphological edge detector with the original IHS transform. From experimental results, depicted in this paper, the new proposed IHS fusion method produced images of high spatial resolution with minimal spectral distortion compared to the original and adaptive IHS pan sharpening.
\end{abstract}

\section{Keywords:}

Image fusion, Pan sharpening, Hyperspectral image, Intensity-hue-saturation (IHS).

* Msc student, Egyptian Air Forces, mohamedredaismail@yahoo.com

** Lecturer, Egyptian Air Forces, ezfarouk@yahoo.com. 


\section{Introduction:}

Some earth observation satellites provide two types of images: panchromatic image (PAN) of high spatial resolution and a hyperspectral image (HSI) of high spectral resolution. To utilize these different types of image data effectively, a number of image fusion techniques have been developed [1]. Image fusion is combining two or more different types of images to improve the quality of the information. Pan sharpening is a pixel level fusion technique used to increase the spatial resolution of the multi/hyperspectral image while preserving its spectral resolution. Pan sharpening is used in many applications such as: improving geometric correction, enhancing certain features in an image, and improving the classification process. Various pan sharpening methods include: Intensity-hue-saturation (IHS) [2], Principle component analysisbased image fusion (PCA) [3], Wavelet-based image fusion [4], Brovey [5] and P+XS [6]. Each method has different spectral and spatial quality. IHS is a popular tool used for pan sharpening because of its efficiency and perfect results. IHS keeps the same spatial resolution of the PAN image but it produces some distortion in the spectral information. This distortion comes from the change in saturation during fusion. In this paper, we introduce an advanced IHS to minimize this distortion by applying a morphological edge detector as well as an adaptive image coefficient with the original IHS transform.

The paper is organized as follows: IHS is introduced in section 2. In section 3, we present the adaptive IHS and how it is used to improve the spectral resolution. Our proposed advanced IHS is presented in section 4. In section 5, we depicted the experimental results of the advanced IHS compared to both original and adaptive IHS followed by the conclusion in section 6 .

\section{Pan sharpening using Original IHS:}

Originally, the IHS is an image transformation technique that converts a color image from the red, green, and blue (RGB) space into the IHS color space [7]. It permits the separation of spatial information into one single intensity band (I) leaving the spectral information hue $(\mathrm{H})$ and saturation $(\mathrm{S})$. In pan sharpening that utilizes IHS, the intensity band (I) in the IHS space is replaced by the PAN image and then transformed back into the original RGB space together with the previous hue $(\mathrm{H})$ and saturation $(\mathrm{S})$ bands. This results in a fused image with higher spatial resolution which is improved by using the PAN image instead of (I) image. The intensity band (I) is calculated by different methods like Smith's hexacone and triangular models [8] as follows:

Smith's hexacone model $I=\max (R, G, B)$

Triangular models $\quad I=\frac{R+G+B}{3}$ 
It was found that the triangular model is best for fusion processes [8]. Researchers have extended this method for the multispectral and hyperspectral images by using

$I=\sum_{i=1}^{N} \alpha_{i} M_{i}$

Where $\alpha_{\mathrm{i}}=1 / \mathrm{N}$ and $\mathrm{N}$ is the number of bands, $\mathrm{M}_{\mathrm{i}}$ is multispectral or HSI bands. Before fusing the two images, first we upsample the hyperspectral images by a factor of four then we normalize each band of the image to the range [0,1]. After completing the initial steps, we apply a histogram matching of the PAN image P to ensure that the mean and standard deviation of the PAN image and HSI image are within the same range as follows:

$P=\frac{\sigma_{I}}{\sigma_{P}}\left(P-\mu_{P}\right)+\mu_{I}$

Where $\sigma_{I}, \sigma_{p}$ and ${ }_{I}, \quad P$ are the standard deviations and means of intensity image and panchromatic image respectively. Finally, as shown in fig.1 the fused hyperspectral image $\mathrm{F}$ is formed by:

$F_{i}=M_{i}+(P-I)$

This fused IHS hyperspectral image has high spatial resolution but suffers from low spectral resolution due to the change in saturation during the fusion process. Adaptive IHS method [9] is used to produce high spectral resolution by combining two techniques, the image adaptive coefficient $a$ which is used to get the intensity band I and extracting the edges of PAN image then combine it with HSI image.

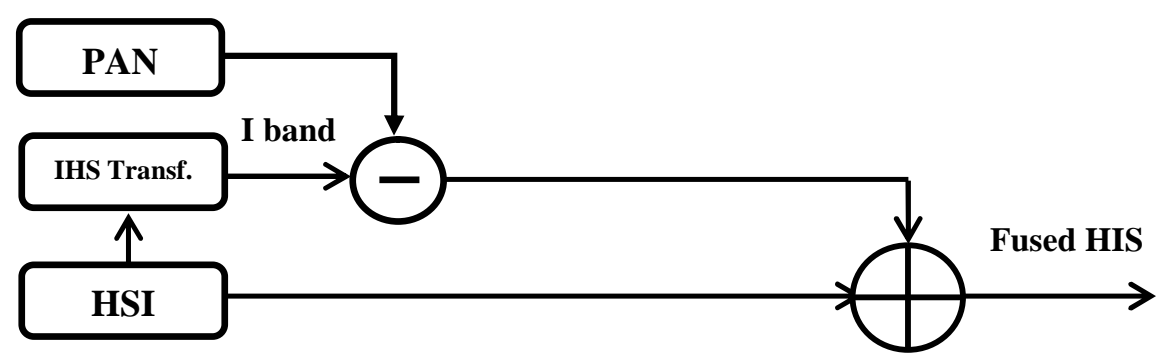

Figure (1): Original IHS Pan Sharpening 


\section{Pan sharpening using adaptive IHS}

Adaptive IHS, shown in fig.2, combines both an adaptive method to compute the image coefficients $\alpha_{i}$ to generate the intensity image I, and an adaptive edge detector which is used to generate a mask to perform fusion at the entire edges only. This combination preserves, to some extent, the spectral resolution of the hyperspectral image and increases its spatial resolution by replacing the intensity image by the PAN image.

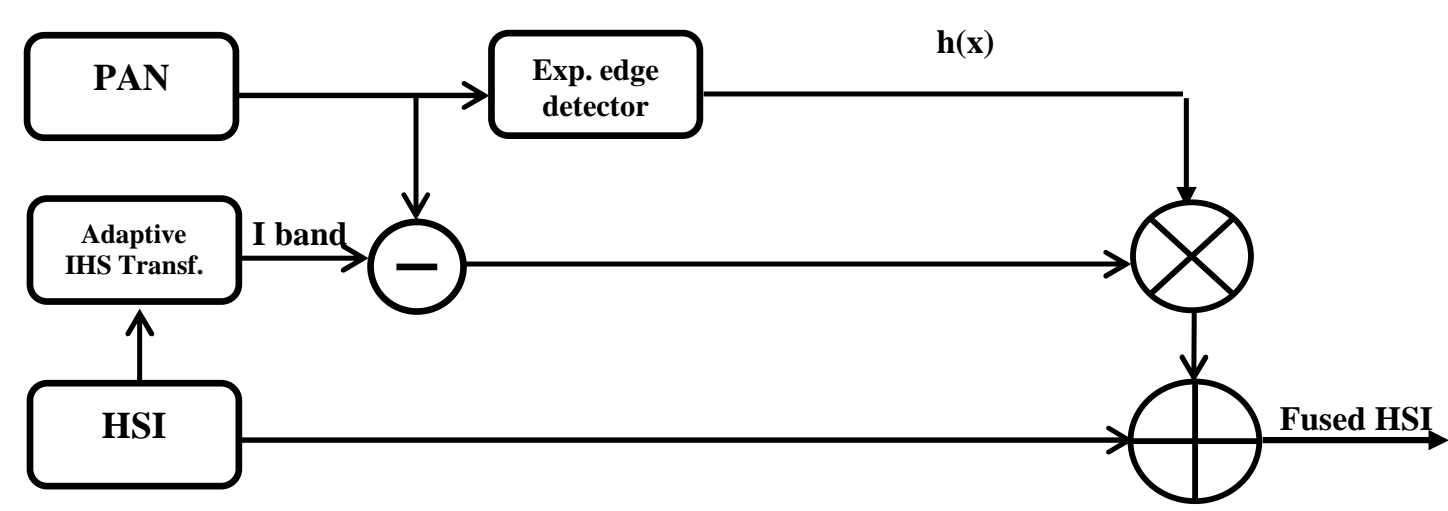

Figure (2): Adaptive IHS Pan Sharpening

\subsection{Adaptive image coefficient method}

In this method, the coefficients which are used in to calculate the intensity band I is computed adaptively. This method approximates the PAN image as closely as possible to the intensity band I so that we can get the value of the coefficients $\alpha_{i}$ for each band of the HSI image as follows:

$P \approx \sum_{i=1}^{N} \alpha_{i} M_{i}$

The following objective function $G(\alpha)$ is used to calculate the coefficients $\alpha_{i}$ :

$\min _{\alpha} G(\alpha)=\sum_{x}\left(\sum_{i}\left(\alpha_{i} M_{i}(x)-P(x)\right)^{2}+\gamma \sum_{i}\left(\max \left(0,-\alpha_{i}\right)\right)^{2}\right.$

The first term guarantees that the coefficients yield a linear combination that approximates the PAN image. The Lagrange multiplier $\gamma$ was added as no negative $\alpha$ is 
required. In order to solve this minimization problem, a gradient descent method and linear algebra methods were used.

\subsection{Adaptive edge detector}

In this method, the edges from the PAN image are extracted to be used as a mask to apply PAN replacement only on the entire edges of the HSI. In other words, the IHS fusion method is applied only where there are edges. The fused multi-channel image $\mathrm{F}$ is calculated by the new formula:

$F_{i}=M_{i}+h(x)(P-I)$

Where $h(x)$ is an edge detecting function with values equal one on edges and equal to zero off edges. The extracted edge can be obtained using standard edge detection methods such as Canny detector [10]. But it was founded experimentally that the best results were produced by the edge detector suggested by Perona and Malik [11]. The edges of the PAN image are extracted using an exponential edge detector, which is calculated by the following formula:

$$
h(x)=\exp \left(-\frac{\lambda}{|\nabla P|^{4}+\varepsilon}\right)
$$

Where $\nabla P$ is the gradient of the PAN image, $\lambda$ is a parameter indicating how large the

gradient should be in order to be an edge and controls the smoothness of the image, and $\varepsilon$ is a small value that enforces a nonzero denominator [12]. The values that proved successful are $\lambda=10^{-9}$ and $\varepsilon=10^{-10}$. Using these values and combining the edge detection with the original IHS, a great modification in the spectral resolution of HSI is achieved.

\section{New Advanced IHS}

This new method based on applying morphological operations on binary images. Morphology is a tool for extracting image components that are useful in the representation and description of region shape such as skeletons and boundaries [13]. Morphological operations apply a structuring element (SE) to an input image and create an output image of the same size. The SE is small compared to the image and its size and shape are defined by a matrix of zeros and ones. The SE is positioned at all possible 
locations in the image and it is compared with the corresponding neighborhood of pixels.

In morphological operation, the value of each pixel in the output image is based on a comparison of the corresponding pixel in the input image with its neighbors. The most basic morphological operations are dilation and erosion. Dilation adds pixels to the boundaries of objects in an image, while erosion removes pixels on object boundaries. The number of pixels added or removed from the objects in an image depends on the size and shape of the structuring element used to process the image.

Skeletonization is an image processing operation that is based on dilation and erosion [14]. It is used to reduce all objects in an image to lines without changing the essential structure of the image. It removes pixels on the boundaries of objects but does not allow objects to break apart. The pixels remaining make up the image skeleton which represents the original objects by a set of lines that can correctly describe the main features like shape and connectivity.

The new advanced IHS is a modified adaptive IHS, where we use a morphological edge detector $h_{\text {new }}(x)$ instead of the exponential edge detector as shown in figure (3).

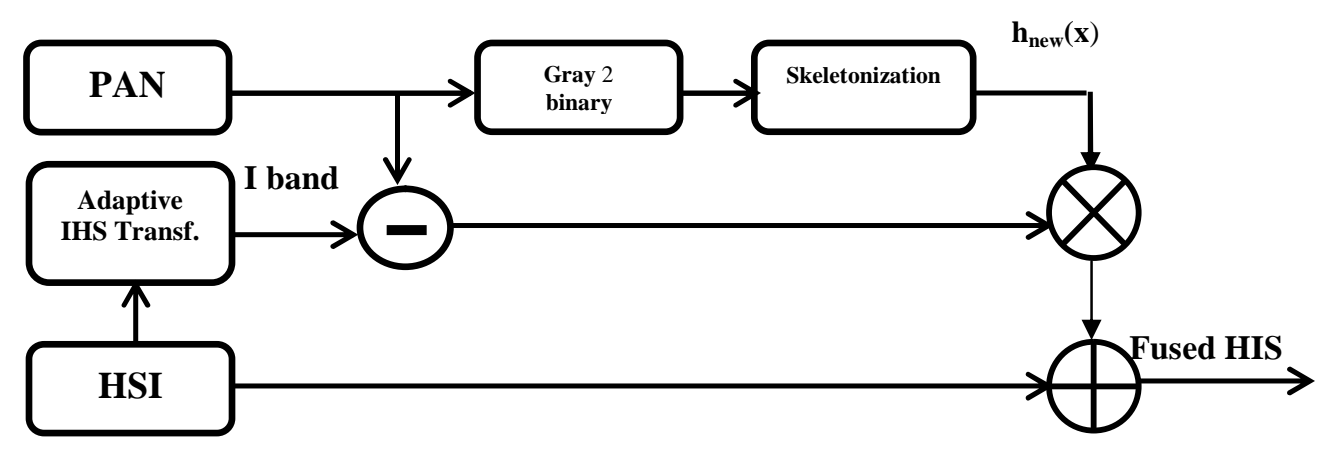

Figure (3): New advanced IHS Pan sharpening

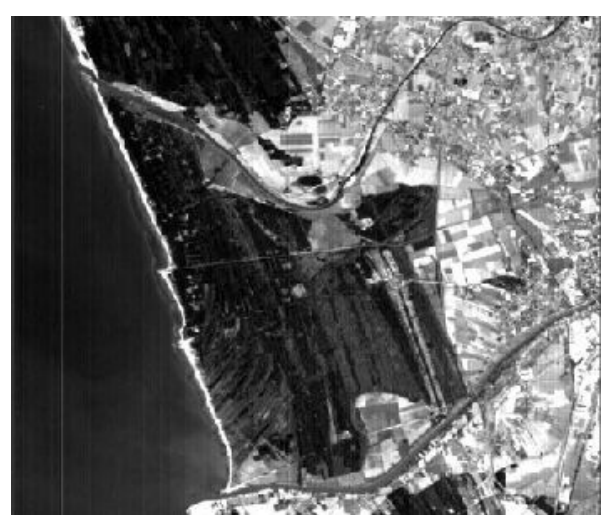

(a)

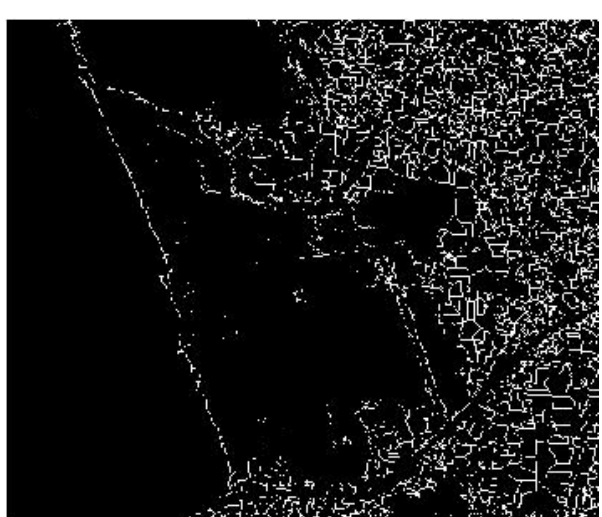

(b) 
Figure (4): Skeletonization of PAN. Image

(a) Original PAN, (b) morphological edges of PAN

First we transfer the PAN image to binary image, and then we apply a skeletonization morphological operation on the binary PAN image. The output of the morphological filter is an image with skeleton of the all object and information in the PAN image. The edges of this new generated image are shown above in figure (4). The output of the morphological filter is the new function $h_{\text {new }}(\mathrm{x})$ which is mentioned above in equation (8). This new modification enhances the spatial quality of the fused IHS image and preserves the spectral resolution.

\section{Experimental Results}

Chris HSI hyperspectral cube and its PAN image data was used for our experiments [15]. The algorithms were implemented in Matlab.

The original IHS, adaptive IHS, and new advanced IHS methods are being compared for their spectral and spatial quality. Spatial quality can be judged visually by seeing the sharpness of the edges, but spectral quality is difficult to notice in this manner. We use several different metrics to help us to analyze our results. Spectral Information Divergence (SID) views each pixel spectrum as a random variable and then measures the discrepancy of probabilistic behaviors between spectra [16]. The relative average spectral error (RASE) characterizes the average performance of the method of image fusion in the spectral bands [17]. We also used the root mean squared error (RMSE) and correlation coefficient (CC) to analyze and compare the spectral quality [18].

The CC calculates the spectral distortion by comparing the $\mathrm{CC}$ between the original hyperspectral bands and the bands of the final fused image. RMSE is the root mean square error between the fused image and the hyperspectral image. Relative dimensionless global error in synthesis (ERGAS) is a normalized version of RMSE designed to calculate the spectral distortion [19].

Also to judge the spatial quality of the pan-sharpened images, we compared the high frequency data from the panchromatic image to the high frequency data from each band of the fused image using a method proposed by Zhou [20]. To extract the high frequency data we apply the following convolution mask to the images:

$$
\operatorname{mask}=\left[\begin{array}{lll}
1 & 1 & 1 \\
1 & 8 & 1 \\
1 & 1 & 1
\end{array}\right]
$$

The resultant filtered images were compared by considering the $\mathrm{CC}$ between each band and the panchromatic image. The closer the average correlation coefficient to one, the 
more closely the edge data of the fused image matches the edge data of the panchromatic, which indicates better spatial quality.

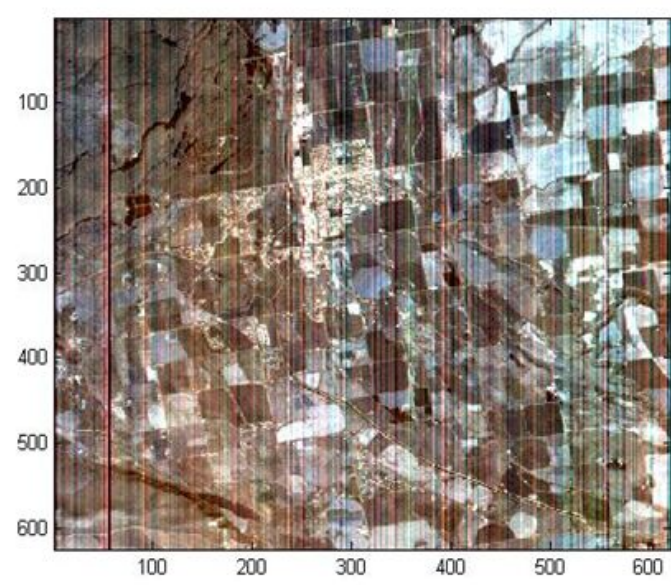

(a)

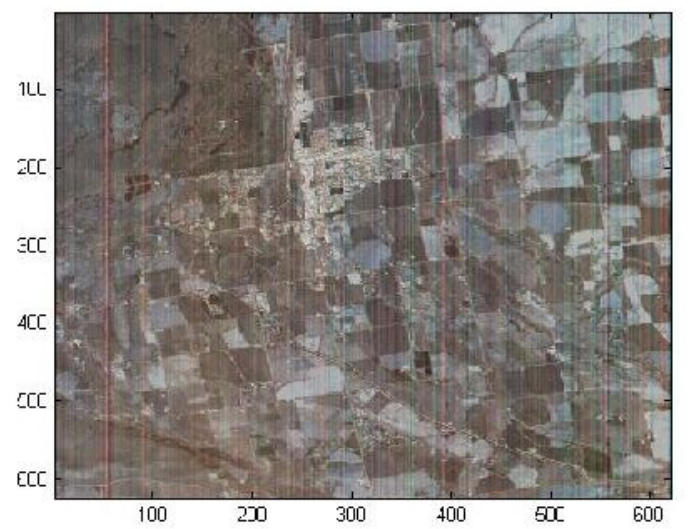

(c)

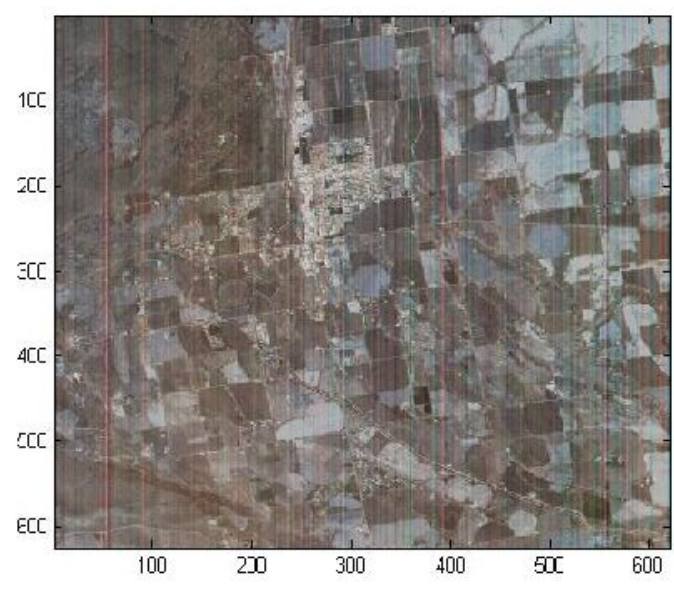

(b)

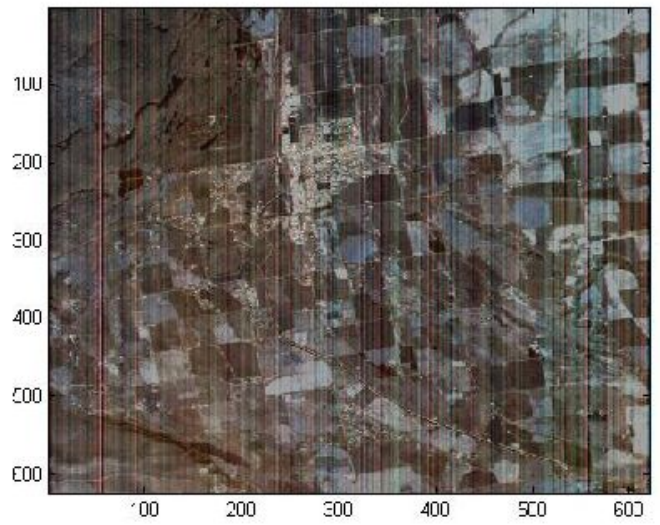

(d)

Figure (5): The HSI image of Chris data Washington 2006-02-06

(a) Original HSI, (b) IHS Fused Image, (c) Adaptive IHS Fused Image, (d) New Advanced IHS Fused Image 


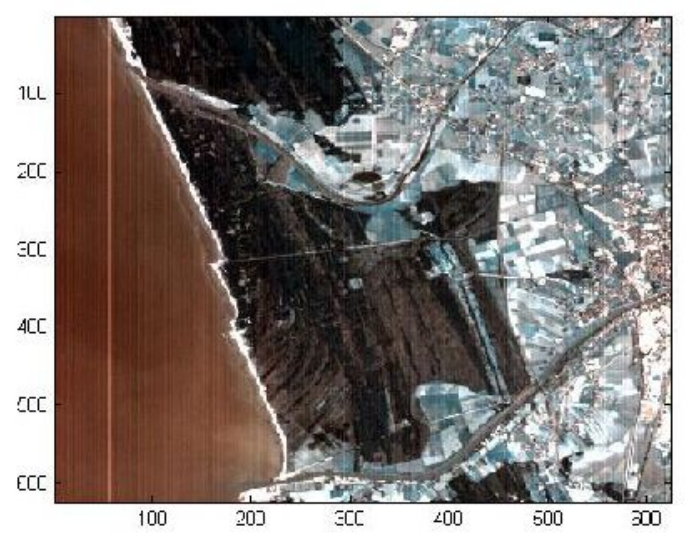

(a)

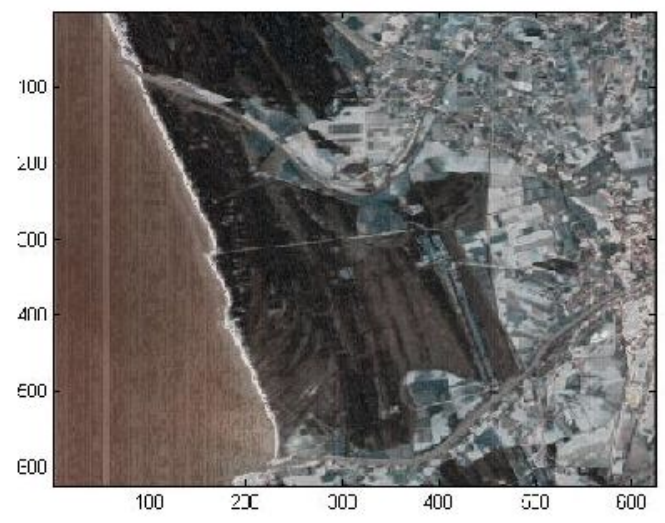

(c)

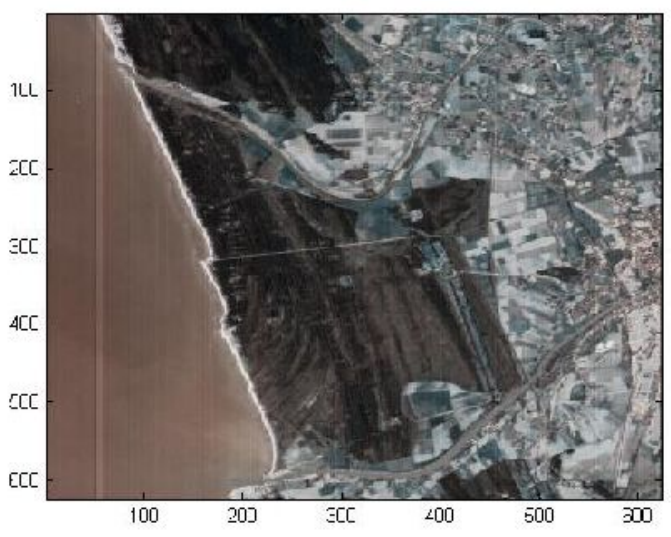

(b)

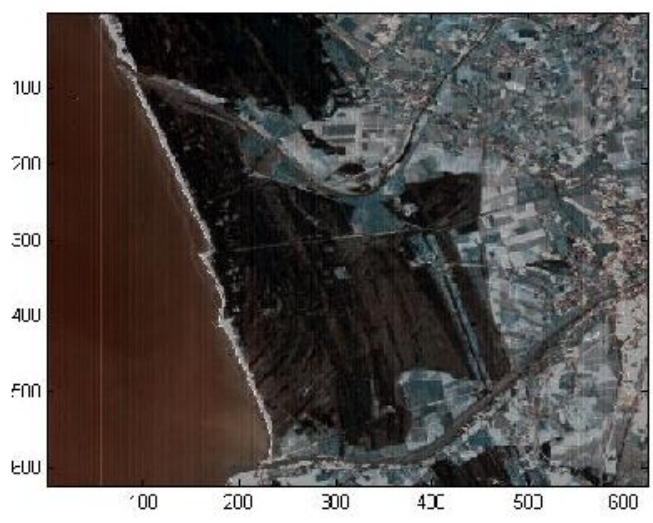

(d)

Figure (6): The HSI image of chris data San-Rossore 2011-03-09 (a) Original HSI, (b) IHS Fused Image, (c) Adaptive IHS Fused Image, (d) New Advanced IHS Fused Image 
Table (1): The spectral and spatial performance analysis metrics for fig. 5.

\begin{tabular}{|c||c|c|c|c|c|c|}
\hline & CC & ERGAS & RASE & RMSE & SID & Spatial \\
\hline Reference value & 0 & 0 & 0 & 0 & 0 & 1 \\
\hline Original IHS & 0.0085874 & 3.9107 & 15.4361 & 18.8054 & 0.16106 & 3.5502 \\
\hline Adaptive IHS & 0.0089951 & 3.8114 & 15.0476 & 18.3321 & 0.16059 & 3.4938 \\
\hline $\begin{array}{c}\text { New Advanced } \\
\text { IHS }\end{array}$ & 0.0026105 & 1.3283 & 5.2683 & 6.4182 & 0.10706 & 1.9492 \\
\hline
\end{tabular}

Table (2): The spectral and spatial performance analysis metrics for fig. 6.

\begin{tabular}{|c||c|c|c|c|c|c|}
\hline & CC & ERGAS & RASE & RMSE & SID & Spatial \\
\hline Reference value & 0 & 0 & 0 & 0 & 0 & 1 \\
\hline Original IHS & 0.19377 & 10.8119 & 40.3082 & 43.5673 & 0.46249 & 3.6238 \\
\hline Adaptive IHS & 0.18113 & 10.4481 & 37.6926 & 40.7402 & 0.45143 & 3.1642 \\
\hline $\begin{array}{c}\text { New Advanced } \\
\text { IHS }\end{array}$ & 0.002371 & 3.022 & 11.3555 & 12.2737 & 0.0012271 & 0.31661 \\
\hline
\end{tabular}

Figures 5 and 6 show the original HSI image and the fused images from the original IHS, the adaptive IHS, and the new advanced IHS fusion methods. The values listed in Tables 1 and 2 correspond to the images in Figures 2 and 3 respectively. In these tables, the values of the metrics are closer to the reference value when using the new advanced IHS fusion method.

\section{Conclusions:}

IHS pan sharpening method gives good spatial quality and is the most commonly used fusion algorithm for its efficiency and simplicity. The lack of spectral quality of the fused HSI resulting from IHS pan sharpening methods was an excellent research field for adapting the original IHS fusion methods. Adaptive IHS is a modified IHS Pan sharpening which combines edge detector as well as adaptive image coefficient method to improve its spectral quality while maintaining its spatial resolution. This enhancement wasn't close enough to the reference values for both spectral and spatial quality. Therefore we proposed a new advanced IHS fusion method that modifies the adaptive IHS by using a morphological edge detector instead of the exponential edge detector, which in turn presents a better spectral quality. The performance evaluation metrics, shown in this paper, confirmed the capability of the new advanced IHS method to get better spatial resolution and minimum spectral distortion. 


\section{References:}

[1] C.Pohl, J.L. Van Genderen, "Multisensor image fusion in remote sensing: concepts, methods and application", International Journal of Remote Sensing, vol. 99, no. 5, pp. 823-854,1998.

[2] T. Tu, S. Su, H. Shyn and P. Huang, "A New Look at IHS-like Image Fusion Methods." Information Fusion, Vol. 2. pp. 177-186. 2001.

[3] V. P. Shah and N. H. Younan. "An Efficient Pan-Sharpening Method via a Combined Adaptive PCA Approach and Contourlets." IEEE Trans. on Geosc. and Remote Sens. 46.2008.

[4] X. Otazu and M. Gonzalez-Ausicana. "Introduction of Sensor Spectral Response Into Image Fusion Methods: Application to Wavelet-Based Methods." IEEE Transactions of Geoscience and Remote Sens. vol. 43, pp. 2376-2385, 2005.

[5] A. Eshtehari and H. Ebadi. "Image Fusion of Landsat ETM+ and Spot Satellite Images Using IHS, Brovey and PCA.” Toosi University of Technology. 2008.

[6] C. Ballester, V. Caselles, L. Igual and J. Verdera. "A Variational Model for P+XS Image Fusion.” International Journal of Computer Vision, pp.43-58, 2006.

[7] M. Choi. "A New Intensity-Hue-Saturation Fusion Approach to Image Fusion with a Tradeoff Parameter." IEEE Transactions of Geoscience and Remote Sensing. Vol. 44, No. 6, pp. 1672-1682,Jun. 2006.

[8] A.R. Smith, "Color gamut transform pairs", Computer graphics, vol.13, no.3, pp.12-19, 1978.

[9] S. Rahmani, M. Strait, D. Merkurjev, et al. "An Adaptive IHS Pan-Sharpening Method", IEEE Geoscience and Remote Sensing Letters, 2010, 7(4): 746-750.

[10] J. Canny. "A Computational Approach to Edge Detection," IEEE Trans. Pattern Anal. Mach. Intell.,vol. PAMI-8, no.6, pp.679-698, Nov. 1986.

[11] P. Perona and J. Malik. "Scale-space and Edge Detection Using Anisotropic Diffusion," IEEE Trans. Pattern Anal. Mach. Intell., vol. 12, no. 7, pp. 629-639, Jul. 1990.

[12] H. Aanaes and J. Sveinsson, "Model-based Satellite Image Fusion," IEEE Trans. Geosci. Remote Sens. Vol. 46, no. 5,

[13] Hyun Geun Yu: Morphological Image Segmentation For Co-Aligned Multiple Images Using Watersheds Transformation, 2004.

[14] P. A. Maragos and R. W. Schafer "Morphological skeleton representation and coding of binary images", IEEE Trans. Acoust., Speech, Signal Processing, vol. 34, pp.1228-1244 1986

[15] www.sstl.co.uk/Downloads/Datasheets/CHRIS-datasheet

[16] C. Chang. "Spectral Information Divergence for Hyperspectral Image Analysis" Proc. Geosci. Remote Sens. Symp. Vol. 1. pp. 509-511, 1999. 
[17] M .Choi, R. Kim. "Fusion of Multispectral and Panchromatic Satellite Images Using the Curvelet Transforms." IEEE Geosci. Remote Sens. Lett. Vol. 2, No. 2, pp. 136-140, Apr. 2005.

[18] Q. Du, O. Gungor, and J. Shan. "Performance Evaluation for Pan-sharpening Techniques." Department of Electrical and computer engineering, Mississippi State University. 24. 2008.

[19] L. Wald. "Quality of High Resolution Synthesized Images: Is There a Simple Criterion?" Proc. Int. Conf. Fusion Earth Data. 2000.

[20] Zhou, J., D.L. Civco, and J.A. Silander, 1998. A wavelet transform method to merge Landsat TM and SPOT panchromatic data, International Journal of Remote Sensing, 19(4):743-757. 